m01.003

\section{Locally controlling the orientation of polymer crystals by nanoimprint lithography}

Zhijun $\mathrm{Hu},{ }^{\text {a,b }}$ Jian Chen, ${ }^{\text {a,b }}$ Vincent Bayot, ${ }^{\text {b,c }}$

Alain M. Jonas ${ }^{\mathrm{a}, \mathrm{b}}$.

${ }^{a}$ Laboratoire de chimie et de physique des hauts polymères (POLY), ${ }^{b}$ Research center in micro- and nanoscopic materials and electronic devices (CeRMiN), ${ }^{c}$ Unité des dispositifs intégrés et circuits electroniques (DICE) Université catholique de louvain, B-1348 Louvain-la-Neuve, Belgium. alain.jonas@uclouvain.be

\section{Keywords: polymer crystals, orientation, nanotechnology}

Polymer crystallization is notoriously difficult to control at a local scale because the growth of polymeric crystals is complicated by the need for cooperative movements of a large number of connected monomers, resulting in a lack of long-range order and rapid loss of orientational correlation over short distances. Typically, polymeric crystals organize into microscale, isotropic, spherical superstructures (spherulites), which are comprised of splaying and branching thin lamellae interleaved with amorphous layers. Here, we report on a fast and easy method to control the orientation of crystals of semicrystalline polymers at the nanometer scale by using nanoimprint lithography [1]. Whenever a hard mold that contains nanoscale features defined on its surface (typical lateral size 50-250 nm; typical height 100 $\mathrm{nm}$ ), is pressed against a molten film of a crystallizable polymer cast on a substrate under controlled temperature and pressure conditions, the molten polymer is forced to flow into the cavities of the mold. The isolation of polymer in separate nano-cavities, which strongly decreases the importance of nucleation by impurities; the confinement of the polymer in nano-sized cavities, which hinders the transport of polymer chains between cavities and controls the fast crystal growth direction; the partial orientation of polymer chains near the vertical walls on the mold due to elongational flow, which eases the formation of nuclei with the crystallographic $\mathrm{c}$ axis parallel to the vertical walls on the mold, lead to a fine control over crystallographic orientation, crystal size and shape of polymer crystals. This is shown for a series of geometries, from simple straight lines to more complex shapes, such as concentric circles and small squares, and for a variety of polymers.

[1] Zhijun Hu et al., Nano Letters 2005, 5, 1738

\section{m01.004}

\section{Microgravity crystal growth in a magnet}

P.W.G. Poodt $^{\mathrm{a}, \mathrm{b},{ }^{*}, \text { M.C.R. Heijna }}{ }^{\mathrm{a}, \mathrm{b}}$, P.C.M. Christianen ${ }^{\mathrm{b}}$, W.J.P. van Enckevort ${ }^{\mathrm{a}}$, W.J. de Grip ${ }^{\mathrm{c}}$, K. Tsukamoto ${ }^{\mathrm{d}}$, J.C. Maan ${ }^{\mathrm{b}}, \mathrm{E}$. Vlieg ${ }^{\mathrm{a}}$

${ }^{a} I M M$, Solid State Chemistry, Radboud university Nijmegen, the Netherlands. ${ }^{b}$ IMM,High Field Magnet Laboratory, Radboud university Nijmegen,the Netherlands. 'Department of Biochemistry, Radboud university Nijmegen, the Netherlands. ${ }^{d}$ Department of Earth and Planetary Science, Tohuku university Sendai, Japan.E-mail: p.poodt@science.ru.nl

\section{Keywords: microgravity, crystal growth, magnetic field}

For protein structure determination using X-ray diffraction, crystallization is often the quality limiting step. During crystal growth, buoyancy driven convection and sedimentation occur under influence of gravity, having a negative effect on crystal quality. Growing crystals in an environment where gravity is reduced or even absent, should reduce these effects and improve crystal quality. A number of crystal growth experiments have been performed in space, but these experiments are rare, extremely expensive and have a low controllability. A promising alternative for crystal growth in space is the use of gradient magnetic fields. At the High Field Magnet Laboratory at the Radboud University Nijmegen, magnetic fields can be produced that counteract gravity, which can be used to grow crystals in an effective microgravity environment. As a model system, we have grown nickel sulphate crystals under such conditions, in order to investigate if the same features occur as during crystal growth in space. We observed an almost complete suppression of convection, an expansion of the depletion zone and a dramatic reduction of the growth rate, as expected for crystal growth in microgravity. These results show that crystal growth in gradient magnetic fields can be a good alternative for microgravity crystal growth in space. Protein crystal growth experiments are in progress. 